\title{
miR-142-5p promotes renal cell tumorigenesis by targeting TFAP2B
}

\author{
MAOSHU ZHU ${ }^{*}$, LIANGNENG ZOU $^{2 *}$, FUHUA LU $^{3}$, LING YE $^{4}$, BIN SU $^{5}$, KAICHUN YANG $^{6}$, \\ MINGHUA LIN ${ }^{7}$, JIANQIAN FU ${ }^{8}$ and YONGWU LI ${ }^{6}$ \\ ${ }^{1}$ The Central Laboratory, Departments of ${ }^{2}$ Geriatrics, ${ }^{3}$ Nephrology, ${ }^{4}$ Respiratory Medicine, ${ }^{5}$ Pharmacy Education, \\ ${ }^{6}$ Emergency, ${ }^{7}$ Pathology, ${ }^{8}$ Medical Oncology, The Fifth Hospital of Xiamen, \\ Xiamen, Fujian 361101, P.R. China
}

Received September 4, 2019; Accepted June 23, 2020

DOI: $10.3892 / \mathrm{ol} .2020 .12187$

\begin{abstract}
The transcription factor AP-2 $\beta(T F A P 2 B)$ serves an important role in kidney development. MicroRNAs (miRNAs) regulate carcinogenic pathways and have gained increasing attention owing to their association with human clear cell renal cell carcinoma (ccRCC) tumorigenesis. However, whether miRNAs could affect renal cell tumorigenesis by regulating $T F A P 2 B$ expression has not been identified. The aim of this study was to investigate the effects of miRNA on $T F A P 2 B$ and its potential role in cell growth, invasion and migration. PCR, western blot and dual luciferase reporter assays were performed to analyze the effects of miR-142-5p on TFAP $2 B$. Furthermore, MTT, flow cytometry, wound healing and Transwell migration assays were used to analyze the effect of miR-142-5p on cell proliferation and migration. The results demonstrated that miR-142-5p targeted TFAP $2 B$ and downregulated the expression of $T F A P 2 B$ at the mRNA and protein levels, promoting cell proliferation and migration in two ccRCC cell lines, 786-O and A-498. This phenomenon supported the theory that miR-142-5p may function as an oncogene in ccRCC. The potential clinical significance of miR-142-5p as a biomarker and a therapeutic target provides rationale for further investigation into miR-142-5p-mediated molecular pathways and how these may be associated with ccRCC development.
\end{abstract}

Correspondence to: Dr Jianqian Fu, Department of Medical Oncology, The Fifth Hospital of Xiamen, 101 Min'an Road, Maxiang, Xiang'an, Xiamen, Fujian 361101, P.R. China

E-mail: fjqsqy@sina.com

Dr Yongwu Li, Department of Emergency, The Fifth Hospital of Xiamen, 101 Min'an Road, Maxiang, Xiang'an, Xiamen, Fujian 361101, P.R. China

E-mail: liyowu@126.com

*Contributed equally

Key words: renal cell carcinoma, transcription factor AP-2 $\beta$, miR-142-5p, proliferation, migration

\section{Introduction}

Clear cell renal cell carcinoma (ccRCC) is a cancer in which malignant cells form in the tubules of the kidney $(1,2)$. ccRCC constitutes $>85 \%$ of kidney cancers, with an estimated 403,262 new cases $(1,3,4)$, and 175,098 deaths in 2018, and the mortality rate is likely to continue to grow $(5,6)$. Although there are several types of standard treatments, nearly half of the diagnosed patients succumb within five years $(3,7)$. Therefore, a better understanding of the molecular mechanisms underlying this disease could help identify new therapeutic targets. In particular, insight into the networks that control signaling cascades associated with cell proliferation and migration may lead to the discovery of novel target genes for ccRCC treatment.

Transcription factor AP-2 $\beta(T F A P 2 B)$ is a member of the TFAP2 protein family, which includes TFAP2-A, -B, -C, -D and $-\mathrm{E}(8)$. Previous studies have revealed that TFAP2 proteins serve as retinoic acid-inducible transcriptional activators and serve important roles in cell growth and differentiation (9-11). A knockout of TFAP $2 B$ enhanced apoptotic renal epithelial cell death in mice (12). However, TFAP2B overexpression has been demonstrated to promote tumor growth, thus, contributing to a poor prognosis in human lung adenocarcinoma (13). In the present study, a systematic investigation of the effects of $T F A P 2 B$ on ccRCC in vitro is described for the first time.

MicroRNAs (miRNAs) are a family of endogenous non-coding single-stranded RNA molecules with a length of 19-22 nucleotides that may act either as tumor suppressors or oncogenes, according to the function of their target genes (14). In the present study it was demonstrated that miRNA (miR)-142-5p serves as an onco-miRNA in ccRCC by targeting $T F A P 2 B$ and downregulating its expression, promoting cell proliferation and migration. Thus, the findings of the present study revealed that the miR-142-5p/TFAP $2 B$ pathway may provide potential therapeutic targets for treatment of ccRCC.

\section{Methods and materials}

Cell lines and cell culture. 293T cells, the human kidney cell line HK-2 and the ccRCC cell lines 786-O and A-498 were obtained from ATCC. All cells were maintained in 
DMEM containing $10 \%$ FBS, $100 \mathrm{U} / \mathrm{ml}$ penicillin, $100 \mathrm{U} / \mathrm{ml}$ streptomycin, and $2 \mathrm{mM} \mathrm{L-glutamine}$ at $37^{\circ} \mathrm{C}$ in a $5 \% \mathrm{CO}_{2}$ atmosphere and $21 \%$ oxygen.

Bioinformatic analysis. The expression of the TFAP $2 B$ was analyzed in The Cancer Genome Atlas (TCGA) ccRCC database (https://www.cancer.gov/about-nci/organization/ccg/research/structural-genomics/tcga). The regulation network of the TFAP $2 B$ and the interaction partners of $T F A P 2 B$ were identified using the STRING (https://string-db.org) software tool. TargetScan screen database (http://www.targetscan. org/vert_72/) was used online to screen the targets and binding sites of miR-142-5p. The miR-142-5p-associated survival was analyzed on the ONCOMIR website (http://www.oncomir. org) (15). The total number of patients for analysis was 517, which was divided into the low expression group $(n=259)$ and the high expression group $(n=258)$. The statistical difference in the survival rate was analyzed by the log-rank test.

Plasmid construction and lentiviral infection. The genomic DNA of 786-O cells was isolated using a FastPure Cell DNA Isolation Mini kit (cat. no. DC102; Vazyme Biotech Co., Ltd.). The 3'UTR of human TFAP2B gene was amplified from the genomic DNA by PCR using $2 X$ Phanta Master Mix (cat. no. P511-01; Vazyme Biotech Co., Ltd.). The primers used are as follows: Forward, 5'-GAGCTCGCTAGCCTCGAGAAA TTTTTAAAAAAAGAAGG-3' and reverse, 5'-CATGCC TGCAGGTCGACTGAAGATGAAAACACAACAATC-3'.

The thermocycling conditions were as follows: $95^{\circ} \mathrm{C}$ for $5 \mathrm{~min}$, followed by 30 cycles of $95^{\circ} \mathrm{C}$ for $15 \mathrm{sec}, 60^{\circ} \mathrm{C}$ for $15 \mathrm{sec}$ and $72^{\circ} \mathrm{C}$ for $60 \mathrm{sec}$. The TFAP2B $3^{\prime} \mathrm{UTR}$ fragment was then cloned downstream to the firefly luciferase reporter gene in the pmirGLO vector (Promega Corporation), generating the pmirGLO-TFAP2B 3'UTR WT plasmid. The mutant TFAP2B 3'UTR was generated using the QuikChange II Site-Directed Mutagenesis kit (Agilent Technologies, Inc.) and was also cloned into the pmirGLO vector, generating the pmirGLO-TFAP2B 3'UTR Mutant plasmid.

The lentivirus-based vector plv-EF1 $\alpha$-PGK-puro (Xiamen Anti-hela Biological Technology Tarde Co., Ltd.) was used to overexpress miR-142-5p. The precursor of miR-142-5p was amplified from the genomic DNA of A- 498 cells by PCR using 2X Phanta Master Mix (cat. no. P511-01; Vazyme Biotech Co., Ltd.) and cloned into the vector. The primers used were as follows: Forward, 5'-TCACGCGTGCGG CCGCAGCCTGAAGAGTACACGCCG-3' and reverse, 5'-CTAGGGATCCGGGCCCGGGCGGGCGGCAGCAGT GGCGTG-3'. The thermocycling conditions were as follows: $95^{\circ} \mathrm{C}$ for $5 \mathrm{~min}$, followed by 30 cycles of $95^{\circ} \mathrm{C}$ for $15 \mathrm{sec}$, $60^{\circ} \mathrm{C}$ for $15 \mathrm{sec}$ and $72^{\circ} \mathrm{C}$ for $30 \mathrm{sec}$. To prepare lentiviral particles, $9 \mu \mathrm{g}$ of plv-EF1 $\alpha$-miR-142-5p-PGK-puro or empty vector plasmid with packaging plasmids $(3 \mu \mathrm{g}$ pMD2G; $6 \mu \mathrm{g}$ pspax2; Xiamen Anti-hela Biological Technology Tarde Co., Ltd.) were co-transfected into $293 \mathrm{~T}$ cells in $10-\mathrm{cm}$ dishes using Lipofectamine ${ }^{\circledR} 2000$ (Invitrogen; Thermo Fisher Scientific, Inc.). Lentivirus-containing medium was collected $48 \mathrm{~h}$ after transfection and used to infect A-498 and 786-O cells $\left(1 \times 10^{6}\right.$ cells/well, in six-well plates) at a multiplicity of infection (MOI) of 30. Two days after lentiviral infection, A-498 and 786-O cells were maintained in the presence of
$1.0 \mu \mathrm{g} / \mathrm{ml}$ puromycin (Sigma-Aldrich; Merck KGaA) for 5 days to generate stable miR-142-5p-overexpressing cells and empty vector negative control cells.

The plasmid pCDH-EF1 $\alpha$-TFAP2B-T2A-BSD for TFAP2B overexpression was constructed using the lentivirus-based vector pCDH-EF1 $\alpha$-MCS-T2A-BSD (Xiamen Anti-hela Biological Technology Tarde Co., Ltd.). Lentiviral particles carrying $T F A P 2 B$ were produced as aforementioned. The cells $\left(1 \times 10^{6}\right.$ cells/well, in six-well plates) stably overexpressing miR-142-5p were infected with the TFAP2B lentivirus at a MOI of 30. Two days after lentiviral infection, the cells were maintained in the presence of $4.0 \mu \mathrm{g} / \mathrm{ml}$ blasticidin $\mathrm{S}$ (Sigma-Aldrich; Merck KGaA) for 10 days to generate A-498 and 786-O cells stably overexpressing both miR-142-5p and TFAP2B (miR-142-5p + TFAP2B).

Transfection. 786-O and A-498 cells were plated into a 6 -well plate $\left(5 \times 10^{5}\right.$ cells/well $)$ at $37^{\circ} \mathrm{C}$. The next day, the pCDH-EF1 $\alpha$-MCS-T2A-BSD plasmid $(4 \mu \mathrm{g} / \mathrm{well})$ and the pCDH-EF1 $\alpha-T F A P 2 B-T 2 A-B S D$ plasmid $(4 \mu \mathrm{g} / \mathrm{well})$ were transfected into the cells using Lipofectamine ${ }^{\circledR} 2000$ (Invitrogen; Thermo Fisher Scientific, Inc.) at $37^{\circ} \mathrm{C}$. After a 24-h period of transfection, the cells were harvested for further experiments.

Reverse transcription-quantitative $P C R \quad(R T-q P C R)$ assay. RNA isolated from cells were subjected to RT using Superscript III Reverse Transcriptase (Invitrogen, Thermo Fisher Scientific, Inc.) at $50^{\circ} \mathrm{C}$ for $30 \mathrm{~min}$. TFAP $2 B$ and $18 \mathrm{~S}$ were transcribed using random RT primers; the miR-142-5p RT primer sequence was 5'-GTCGTATCCAGTGCAGGG TCCGAGGTATTCGCACTGGATACGACAGTAGT-3' and the U6 RT primer sequence was 5'-CGCTTCACGAATTTG CGTGTCAT-3'. qPCR was conducted using a Bio-Rad CFX96 system with a ChamQ SYBR ${ }^{\circledR}$ qPCR Master Mix kit (Vazyme Biotech Co., Ltd.) to determine the mRNA or miRNA expression levels of the genes of interest. The method of quantification was as described previously (16). The thermocycling conditions were as follows: $94^{\circ} \mathrm{C}$ for $3 \mathrm{~min}$, followed by 40 cycles of $94^{\circ} \mathrm{C}$ for $15 \mathrm{sec}, 60^{\circ} \mathrm{C}$ for $20 \mathrm{sec}$ and $72^{\circ} \mathrm{C}$ for $20 \mathrm{sec}$. Each detection was performed in triplicate. Expression levels were normalized to those of U6 or 18S ribosomal RNA.

The following primers were used for qPCR: miR-142-5p forward, 5'-GCG CGA ACATAA AGT AGA A AG C-3' and reverse, 5'-AGTGCAGGGTCCGAGGTATT-3'; U6 forward, 5'-GCTTCGGCAGCACATATACTAAAAT-3' and reverse, 5'-CGCTTCACGAATTTGCGTGTCAT-3'; TFAP2B forward, 5'-GTTGAAGATGCCAATAACAGCGG-3' and reverse, 5'-GGACGGAGCAAAACACCTCGC-3'; and 18S forward, 5'-CGACGACCCATTCGAACGTCT-3' and reverse, 5'-CTCTCCGGAATCGAACCCTGA-3'.

Western blotting. Cells were lysed in ice-cold RIPA buffer (Sangon Biotech Co., Ltd.) and the protein was quantified using a BCA Protein Assay kit (Abcam). Lysates (20 $\mu \mathrm{g} / \mathrm{sample})$ were loaded on 8-12\% denaturing SDS-PAGE gels and transferred to a PVDF membrane (Roche Diagnostics GmbH). Tris- $\mathrm{HCl}$ buffer containing $5 \%$ bovine serum albumin (BSA; Beijing Solarbio Science \& Technology Co., Ltd.) was used to block the membranes at $28^{\circ} \mathrm{C}$ for $2 \mathrm{~h}$. The membrane was 
probed with the primary antibodies prepared in Tris- $\mathrm{HCl}$ buffer containing 5\% BSA at $4{ }^{\circ} \mathrm{C}$ overnight. Subsequently, the membranes were washed three times with Tris- $\mathrm{HCl}$ buffer containing $0.1 \%$ Tween-20, followed by incubation with the appropriate secondary antibodies prepared in Tris- $\mathrm{HCl}$ buffer at $28^{\circ} \mathrm{C}$ for $1 \mathrm{~h}$. Finally, the membranes were washed three times, detected and visualized by an enhanced chemiluminescence detection system (Thermo Fisher Scientific, Inc.). The antibodies used in this study included anti-TFAP2B (1:500; cat. no. 13183-1-AP; ProteinTech Group, Inc.), anti-GAPDH (1:5,000; cat. no. YM3029; ImmunoWay Biotechnology Company), HRP-conjugated goat anti-rabbit IgG (1:1,000; cat. no. 7074; Cell Signaling Technology, Inc.) and HRP-conjugated rabbit anti-mouse $\operatorname{IgG}$ (1:1,000; cat. no. 7076; Cell Signaling Technology, Inc.).

Dual luciferase reporter assay. miR-142-5p mimics (5'-CAU AAAGUAGAAAGCACUACU-3') and negative control mimic (mimics ctrl; 5'-UUGUACUACACA AAAGUA CUG-3') were obtained from Guangzhou RiboBio Co., Ltd. $293 \mathrm{~T}$ cells were seeded into a 6 -well plate $\left(10^{6}\right.$ cells/well $)$ and transfected with mimics ctrl or miR-142-5p mimics (200 pmol/well), along with the pmirGLO-WT TFAP2B 3'UTR (4 $\mu \mathrm{g} /$ well) or pmirGLO-Mutant TFAP2B 3'UTR plasmid (4 $\mu \mathrm{g} /$ well) using Lipofectamine ${ }^{\circledR} 2000$ (Invitrogen; Thermo Fisher Scientific, Inc.), and kept at $37{ }^{\circ} \mathrm{C}$. The cells were lysed at $48 \mathrm{~h}$ post-transfection and luciferase activity was measured using the Dual-Glo Luciferase Assay System (Promega Corporation) according to the manufacturer's instructions. The firefly luciferase activity was calibrated to Renilla luciferase activity. Each treatment was carried out in triplicate.

Cell proliferation assay. A total of $3 \times 10^{3}$ cells/well were seeded onto 96 -well plates. Cell proliferation was measured using a MTT cell proliferation assay kit (cat. no. 11465007001; Shanghai Qcbio Science \& Technologies Co., Ltd.) at each time point $(0,24,48$ and $72 \mathrm{~h})$ for $1 \mathrm{~h}$ at $37^{\circ} \mathrm{C}$. The absorbance was measured at $450 \mathrm{~nm}$. All experiments were performed in triplicate.

Cell cycle assay. A total of $1 \times 10^{6}$ cells/well were seeded onto 6-well plates. Next day, 786-O and A-498 cells were harvested and fixed in $70 \%$ ethanol at $4{ }^{\circ} \mathrm{C}$ overnight. The fixed cells were then incubated with PBS containing $10 \mu \mathrm{g} / \mathrm{ml}$ RNase A (Sangon Biotech Co., Ltd.) and 0.2\% Triton X-100 for $30 \mathrm{~min}$ at $37^{\circ} \mathrm{C}$, and then stained with $20 \mu \mathrm{g} / \mathrm{ml}$ propidium iodide for $30 \mathrm{~min}$ in the dark at room temperature. The stained cells were analyzed using the NovoCyte flow cytometer with NovoCyte 1.4.1 software (ACEA Biosciences, Inc.). All experiments were performed in triplicate.

Wound healing assay. 786-O and A-498 cells with stable overexpression of TFAP2B were seeded into 6-well plates to reach $100 \%$ confluence. Wound was generated using a $200 \mu \mathrm{l}$ pipette tip with a straight scratch. Cells were maintained in serum free medium for $24 \mathrm{~h}$ and were observed at x40 magnification under a bright field microscope (Motic Incoporation, Ltd.). The percentage of the wound healing was quantified by ImageJ 1.8.0 software (National Institutes of Health).
Transwell assay. Migration assays were performed using Transwell plates with $8-\mu \mathrm{m}$ pore size membranes. A total of $2.5 \times 10^{5} 786-\mathrm{O}$ and $\mathrm{A} 498$ cells with stable overexpression of TFAP2B were plated in the upper chambers of the Transwell plates. After a $24-\mathrm{h}$ period of incubation at $37^{\circ} \mathrm{C}$, the migrated cells were stained with $0.5 \%$ toluidine blue and photographed at X100 magnification under a bright field microscope (Motic Incoporation, Ltd.). The migrated cells were counted using Image $\mathrm{J}$ in three random fields.

Statistical analysis. All statistical analyses were conducted using SPSS version 19.0 (IBM Corp.) and GraphPad Prism version 8.0 (GraphPad Software, Inc.). Data are presented as the mean \pm SD. Differences between two groups were analyzed with Student's t-test, whereas ANOVA followed by Tukey's post-hoc test was used for multiple comparisons of three or more experimental groups. $\mathrm{P}<0.05$ was considered to indicate a statistically significant difference.

\section{Results}

Database analysis of the expression profile of TFAP $2 B$ and its regulation. To identify genes that were lowly expressed in $\mathrm{RCC}$, a screen was conducted using Oncomine software. The $T F A P 2 B$ expression level in ccRCC was decreased compared with that in the normal tissue group; thus, TFAP $2 B$ was selected for further experiments based on the P-value and median expression rank (Fig. 1A). It is possible that TFAP2B protein levels may be regulated by miRNAs, as it contains a conserved miRNA target site in the $3^{\prime}$ UTR region (Fig. 1B). To confirm this hypothesis and to determine the possible miRNAs that may target TFAP2B, a screen using the TargetScan software was performed, which lead to the identification of miR-142-5p as a putative miRNA that can bind to TFAP2B 3'UTR regulating its protein expression (Fig. 1C). Furthermore, TCGA ccRCC database analysis demonstrated that high levels of miR-142-5p is negatively associated with lower TFAP $2 B$ mRNA expression in patient samples (Fig. S1A and B). Moreover, the miR-142-5p expression was significantly associated (log-rank test) with the hazard ratio of death status in various cancer types in the TCGA public database using Kaplan-Meier analysis by long-rank tests between the miR-142-5p high expression group and the miR-142-5p low expression group (Fig. S1C). And the expression level of miR-142-5p was significantly associated with the survival rate in the kidney cancer (Fig. S1C). Furthermore, the STRING software tool was used to analyze the TFAP2B interaction network, which demonstrated that the $\mathrm{Cbp} / \mathrm{p} 300$-interacting transactivator with ED-rich tail family, the TF2P family, the small ubiquitin-like modifier family and TP53 might be important regulators in the expression and functions of TFAP2B due to their direct and stronger interactions (Fig. S1D).

Comparison of TFAP $2 B$ expression levels in normal kidney cells and renal tumor cells. To identify suitable ccRCC cell lines for further study, RT-qPCR and western blot assays were used to analyze mRNA and protein expression levels of TFAP2B in ccRCC cell lines compared with the normal kidney cell line HK-2. As shown in Fig. 2A, the results indicated that the mRNA expression levels of TFAP $2 B$ was lower in both 
A

Median rank P-value Gene

$320 \quad 250 \times 10^{-30}$ TFAP2B

$39.0 \quad 1.74 \times 10^{-6}$ CALB1

$46.0 \quad 5.70 \times 10^{-29}$ TMPRSS

48.0

61.0

81.0

82.0

86.0

90.0

90.5

95.0

98.0

98.0

104.0

106.0

111.0

114.0

133.0

134.0

137.0

$8.61 \times 10^{-9}$ SLC12A

$7.17 \times 10^{-10}$ PTH1R

$3.08 \times 10^{-9}$ CLIC5

$2.50 \times 10^{-7} \quad$ AlF1L

$2.50 \times 10^{-8}$ NPHS2

$2.50 \times 10^{-6}$ GPC3

$2.50 \times 10^{-6}$ Clorf226

$2.50 \times 10^{-27}$ ELF5

$2.50 \times 10^{-9}$ ERBB4

$2.50 \times 10^{-6}$ SFRP1

$2.50 \times 10^{-6} \quad \mathrm{KCNJ} 1$

$2.50 \times 10^{-9}$ TFCP2L1

$2.50 \times 10^{-6}$ ALAD

$2.50 \times 10^{-6}$ GATA3

$2.50 \times 10^{-7}$ ACPP

$2.50 \times 10^{-6}$ SORCS1

$2.50 \times 10^{-5} \quad$ LPPR 1
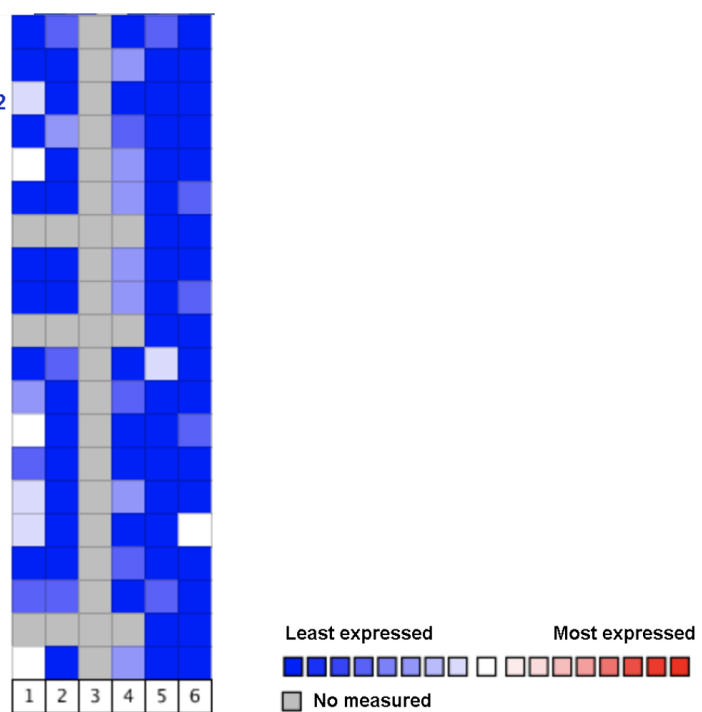

Legend

1. Clear Cell Sarcoma of the Kidney vs. 4. Clear Cell Renal Cell Carcinoma vs. Normal

Normal

Cutcliffe Renal, Clin Cancer Res, 2005

2. Clear Cell Renal Cell Carcinoma vs. Normat

Gumz Renal, Clin Cancer Res, 2007

3. Clear Cell Renal Cell Carcinoma vs.
Higgins Renal, Am J Pathol, 2003

Jones Renal, Clin Cancer Res, 2005

5. Clear Cell Renal Cell Carcinoma vs. Norma

Lenburg Renal, BMC Cancer, 2003

6. Clear Cell Renal Cell Carcinoma vs. Norma

Yusenko Renal, BMC Cancer, 2009

B

\begin{tabular}{|c|c|}
\hline & TFAP2B 3'UTR \\
\hline & 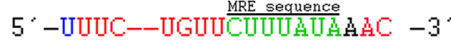 \\
\hline & $5^{\prime}-\mathrm{UUUUUU}$ C- - \\
\hline sus & CUUUAUAAAAC -3 \\
\hline ouse & EUUUUA AUA $G A C-3$ \\
\hline & -UUUC-OUGUUCUUUAUAAGAC - $3^{\prime}$ \\
\hline ow & $5^{\circ}$-UUUC--UGUUCUUUA \\
\hline $\mathrm{Pi}$ & $5^{\circ}$-UUUUC-DJGUUCUUUAUAAGAC -3 \\
\hline & 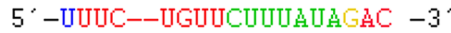 \\
\hline
\end{tabular}

C

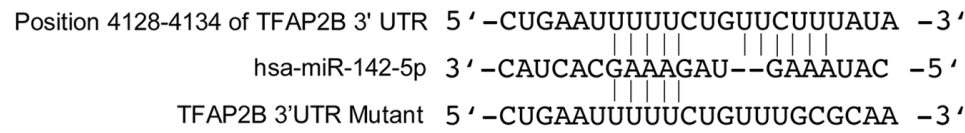

Position 4128-4134 of TFAP2B 3' UTR 5’-CUGAAUUUUUCUGUUCUUUAUA - 3 ‘

hsa-miR-142-5p 3'-CAUCACGAAAGAU--GAAAUAC -5 '

TFAP2B 3'UTR Mutant 5 '-CUGAAUUUUUCUGUUUGCGCAA -3 ’

Figure 1. TFAP $2 B$ expression appears to be downregulated by miR-142-5p in a bioinformatic analysis. (A) The expression levels of $T F A P 2 B$ is lower in clear cell renal carcinoma compared with expression in normal tissue. (B) The conserved miRNA sites in TFAP2B. (C) miR-142-5p target site in the 3'UTR of $T F A P 2 B$. miR/miRNA, microRNA; MRE, miRNA response element; $T F A P 2 B$, transcription factor AP- $2 \beta$; UTR, untranslated region.

renal tumor cells 786-O and A498 compare with expression HK-2. In addition, TFAP $2 B$ protein expression levels were lower in the ccRCC cell lines compared with HK-2 (Fig. 2B). These results suggested that 786-O and A498 cells can recapitulate the effects observed with Oncomine software screen, and therefore were selected as a suitable model for subsequent studies.

miR142-5p targets TFAP2B. Based on the TargetScan screen database analysis, the interaction between miR-142-5p and its putative target TFAP $2 B$ was examined (Fig. 1B). The results demonstrated that the miR-142-5p mediated-decrease of TFAP2B protein level was rescued by the TFAP2B overexpression vector, compared with miR-142-5p group (Fig. S2A and B). In addition, as shown in Fig. S2C and D, TFAP2B overexpression vector could induce the overexpression of TFAP2B alone, compared with the empty vector negative control cells (Fig. S2A and B). Furthermore, a dual luciferase reporter assay revealed a significant decrease in the luciferase activity in 293T cells co-transfected with miR-142-5p and the
TFAP2B-3'UTR WT luciferase reporter vector compared with the luciferase activity in cells co-transfected with the negative control (mimics ctrl) and the reporter vector. Besides, the TFAP2B-3'UTR Mutant abolished the interaction between the miR-142-5p and the TFAP2B-3'UTR region as no significant differences were observed in the luciferase activity between miR-142-5p and mimics control (Fig. 2C). Moreover, the effects of miR-142-5p overexpression on TFAP $2 B$ in 786-O and A-498 cell lines were examined by RT-qPCR and western blot assays. As shown in Fig. 2D, the level of miR-142-5p was higher in miR-142-5p-overexpression (OE) 786-O and A-498 cells compared with expression levels in the negative control group. TFAP $2 B$ mRNA and protein levels were markedly decreased in miR-142-5p-OE cells (Fig. 2E and F, respectively). Based on the above results, it was concluded that miR142-5p targeted $T F A P 2 B$ and suppressed its expression in these cell lines.

miR142-5p promotes proliferation of ccRCC cells. To study the effects of the miR142-5p on ccRCC cell proliferation, 
A

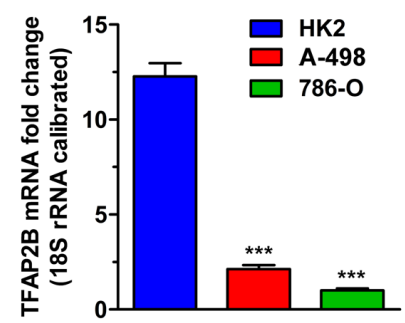

D

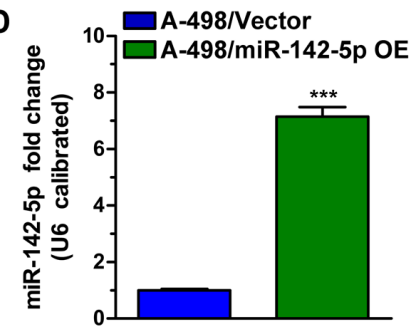

E

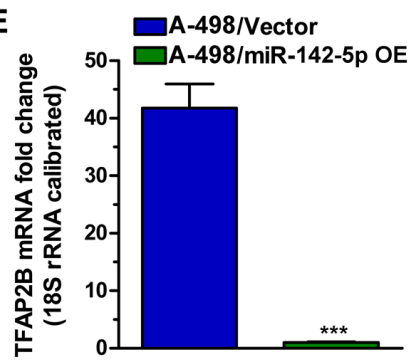

B
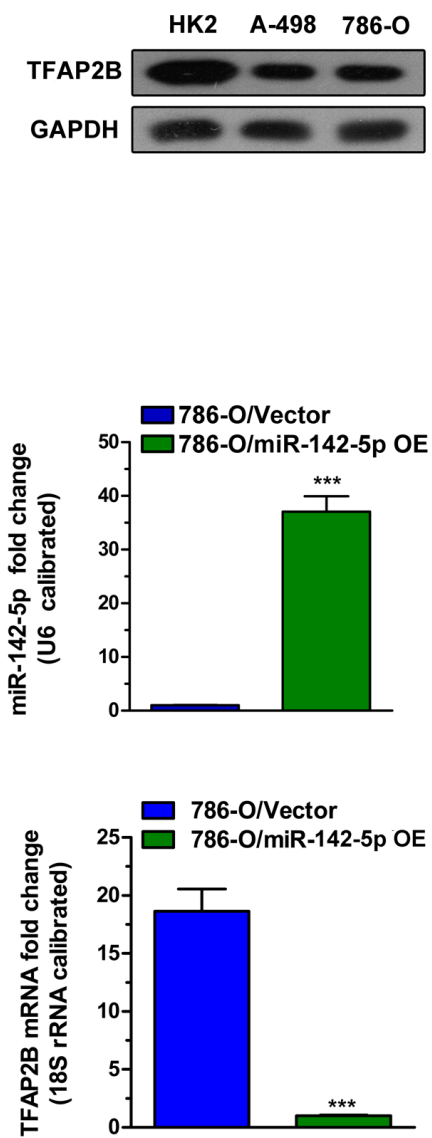

C

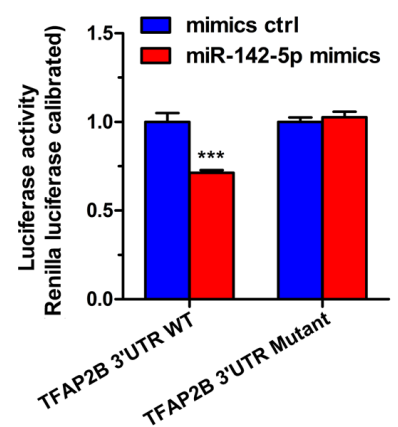

$\mathbf{F}$
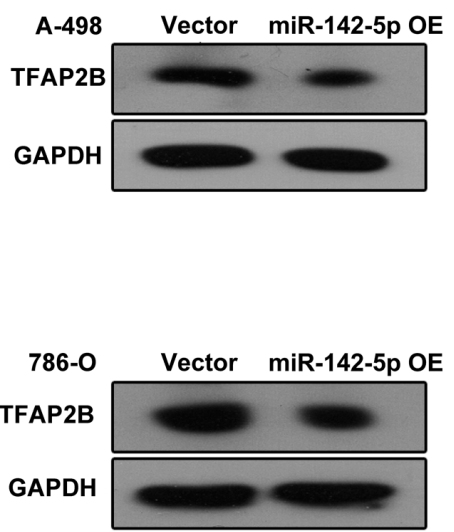

Figure 2. RT-qPCR and western blotting assay. (A) Comparison of the relative mRNA expression levels of TFAP2B between HK-2 normal kidney cells and ccRCC cells, A-498 and 786-O, as measured by RT-qPCR. The HK-2 was used as a comparison control. ${ }^{* * *} \mathrm{P}<0.001$ vs. HK2. (B) TFAP2B protein expression levels in HK-2 cells and ccRCC cells A-498 and 786-O were determined by western blot analysis. (C) Dual-luciferase reporter assay showing reduced luciferase reporter activity in $293 \mathrm{~T}$ cells containing the TFAP2B-3'UTR WT fragment. ${ }^{* * *} \mathrm{P}<0.001$ vs. mimics control. (D) miR-142-5p overexpression was detected by RT-qPCR in both A-498 and 786-O cells. ${ }^{* * *} \mathrm{P}<0.001$ vs. vector. (E) The mRNA level of TFAP2B was detected by the RT-qPCR for different groups. ${ }^{* * *} \mathrm{P}<0.001$ vs. vector. (F) Image from the western blot analysis in the miR142-5p-OE or control group demonstrating the TFAP2B protein expression levels in A-498 and in 786-O cells. Data are presented as the mean \pm SD; One-way Anova tests was used. miR, microRNA; NC, negative control; OE, overexpression; ccRCC, clear cell renal cell carcinoma; RT-qPCR, reverse transcription-quantitative PCR; TFAP2B, transcription factor AP-2 $\beta$; UTR, untranslated region.

the proliferation abilities of 786-O and A-498 cell lines overexpressing miR-142-5p or co-overexpressing miR-142-5p + TFAP2B were evaluated with an MTT assay (Fig. 3A). An increased proliferation rate was observed in miR-142-5p-OE cells compared with the empty vector negative control group in each cell line. In addition, the overexpression of TFAP $2 B$ could eliminate the miR142-5p-induced increase in cell proliferation (Fig. 3A).

Because miR142-5p promoted 786-O and A-498 cell proliferation, we evaluated the effects of miR 142-5p on 786-O and A-498 cell cycle progression by flow cytometry. The results demonstrated that the percentage of cells in phase $\mathrm{G}_{0} / \mathrm{G}_{1}$ was significantly lower in miR-142-5p-OE 786-O and A-498 cells, whereas the proportion of $S$ phase cells was significantly higher in miR-142-5p-OE 786-O and A-498 cells compared with the negative control group (Fig. 3B and $\mathrm{C}$ ). This effect was eliminated by $T F A P 2 B$ overexpression, which lead to an increased percentage of A-988 cells in phase $G_{0} / G_{1}$ and reduced the percentage of 786-O cells in $\mathrm{S}$ phase. These results indicated that miR $142-5 p$ could prevent $G_{1}$ phase arrest in 786-O and A-498 cells and significantly promote ccRCC cell proliferation.
miR142-5p enhances migration of ccRCC cells. Results from wound healing and Transwell migration assays demonstrated that miR-142-5p-OE 786-O and A-498 cells exhibited significantly increased cell migration compared with the negative control cells (Figs. 3D and E and 4). As expected, overexpression of TFAP2B significantly removed the influence of increased miR142-5p expression on cell migration (Fig. 3E). These data suggested that the migratory ability of ccRCC cells was enhanced by miR-142-5p but was weakened by TFAP $2 B$.

\section{Discussion}

In the present study, $T F A P 2 B$ expression was found to be markedly low in renal cancer, indicating that TFAP $2 B$ might function as a tumor suppressor in ccRCC. miR-142-5p was found to directly target $T F A P 2 B$ and downregulate its expression, indicating that miR-142-5p may act as an oncogenic microRNA. Finally, the effect of miR-142-5p and TFAP $2 B$ on ccRCCs was systematically investigated through a series of cell function experiments. The results demonstrated that miR-142-5p increased cell proliferation and migration and that $T F A P 2 B$ could reverse these effects. 
A

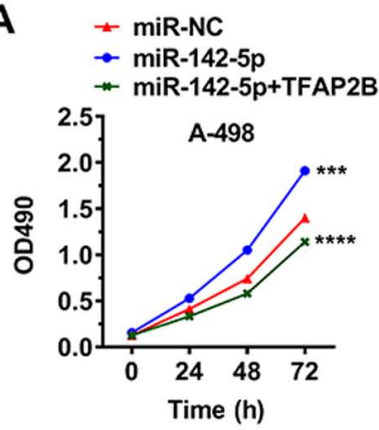

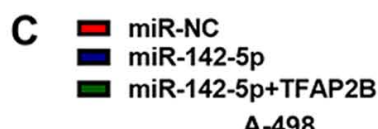

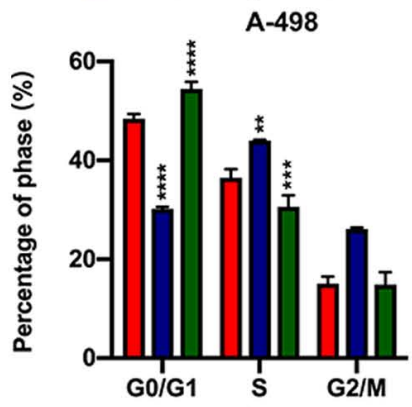

D
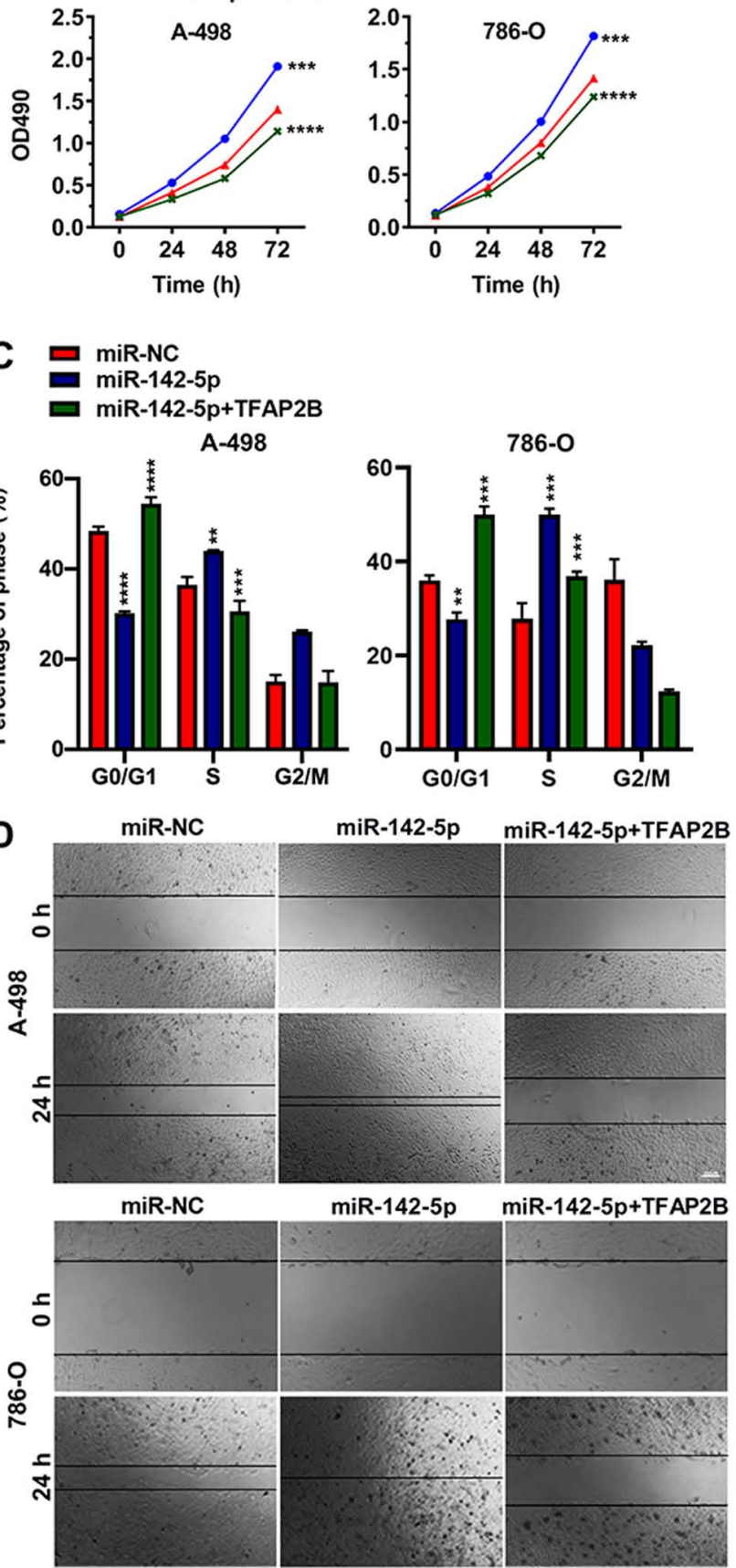
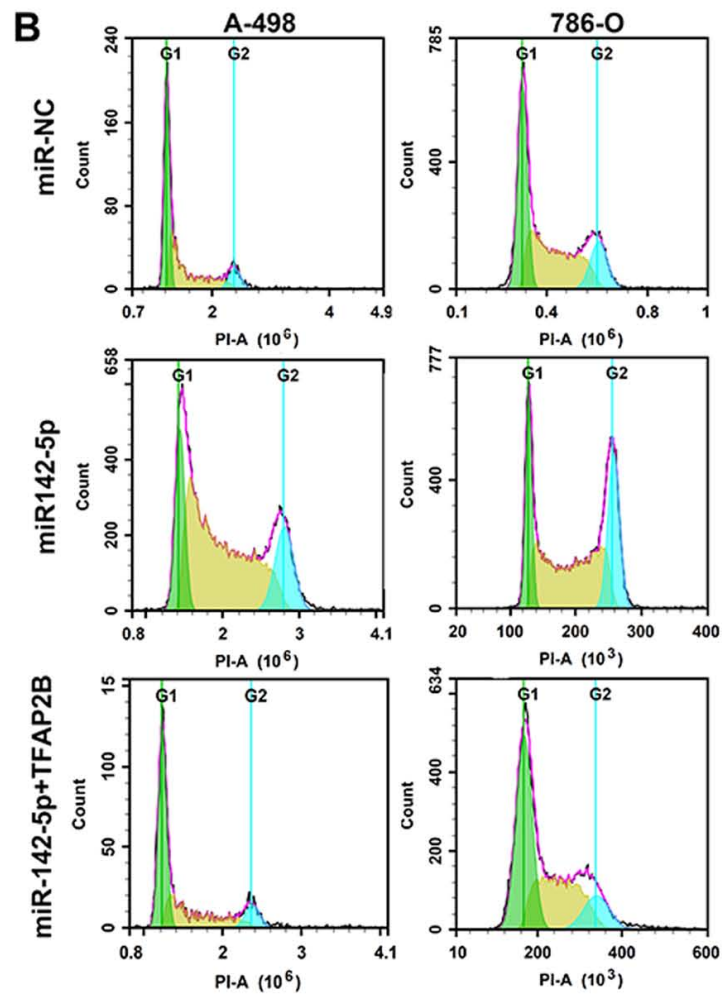

E

A-498

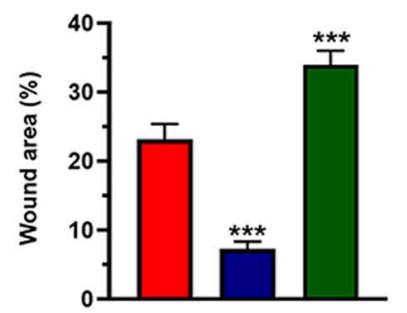

$\square$ miR-NC

miR-142-5p

$\square$ miR-142-5p+TFAP2B

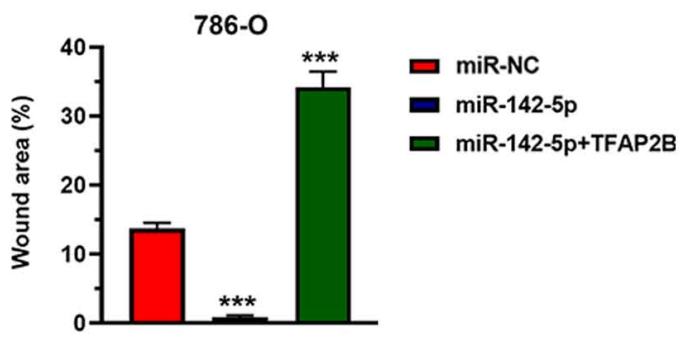

Figure 3. miR-142-5p promotes cell proliferation and migration. (A) MTT cell proliferation assay in 786-O and A-498 cells overexpressing miR-142-5p alone or in combination with TFAP $2 B$ overexpression. (B) Representative images of cell cycle analysis by flow cytometry (C) Percentage of cells in different stages of the cell cycle in the different groups. (D) Representative images of the wound healing assay in cells transfected with miR-142-5p, miR-142-5p and TFAP2B or negative control. (E) Statistical analysis of the wound healing assay. Data are presented as the mean $\pm \mathrm{SD} ;{ }^{* * *} \mathrm{P}<0.01 ;{ }^{* * * *} \mathrm{P}<0.001 ;$ and ${ }^{* * * *} \mathrm{P}<0.0001 \mathrm{vs}$. miR-NC. miR, microRNA; NC, negative control; OD, optical density; TFAP2B, transcription factor AP-2 $\beta$.

Cell cycle assays showed that overexpression of miR-142-5p reduced the population of $\mathrm{G}_{0} / \mathrm{G}_{1}$ phase cells, which indicated that miR-142-5p may promote cell growth by forcing them to enter the $\mathrm{S}$ phase.

A miRNA can target numerous genes and act as either a tumor suppressor or oncogenic miRNA, depending on the function of targeted genes. Liu et al (17), reported that miR-142-5p promotes development of colorectal cancer by targeting succinate dehydrogenase complex iron sulfur subunit B.
However, a study by Wang et al (18) showed that miR-142-5p targets PI3K- $\alpha$ to suppress tumorigenesis in non-small cell lung cancer. In human osteosarcoma, miR-142-5p also suppresses proliferation and promotes apoptosis by targeting phospholipase A and acyltransferase 3 (19). In the present study, miR-142-5p was shown to promote proliferation and migration of ccRCC cells by targeting TFAP $2 B$, which is consistent with study results reported by Liu et al (20) that revealed that miR-142-5p promotes cell growth and migration 
A
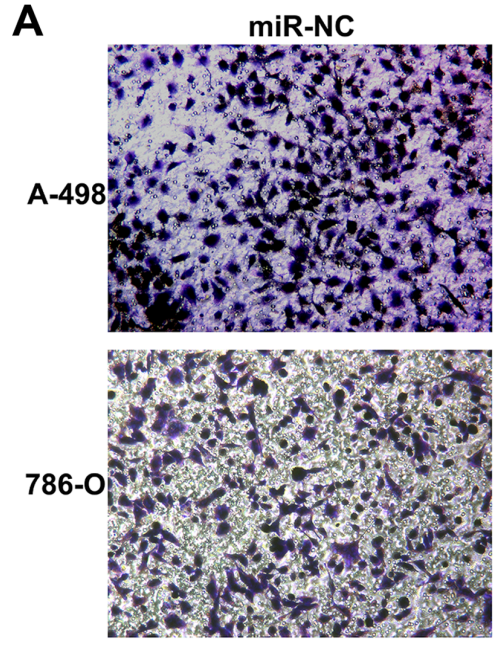

miR-142-5p
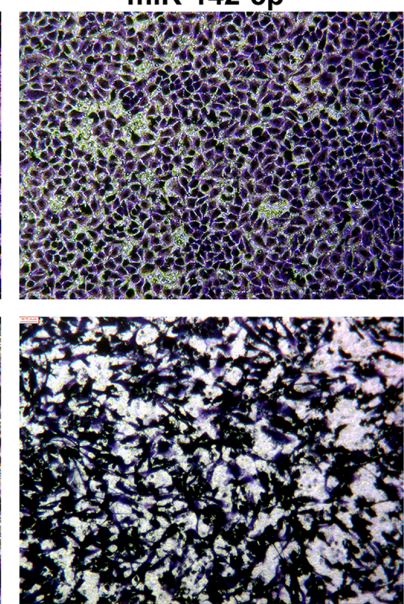

miR-142-5p+TFAP2B
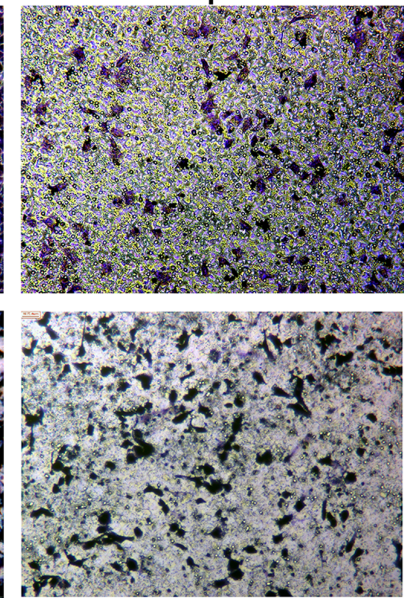

B
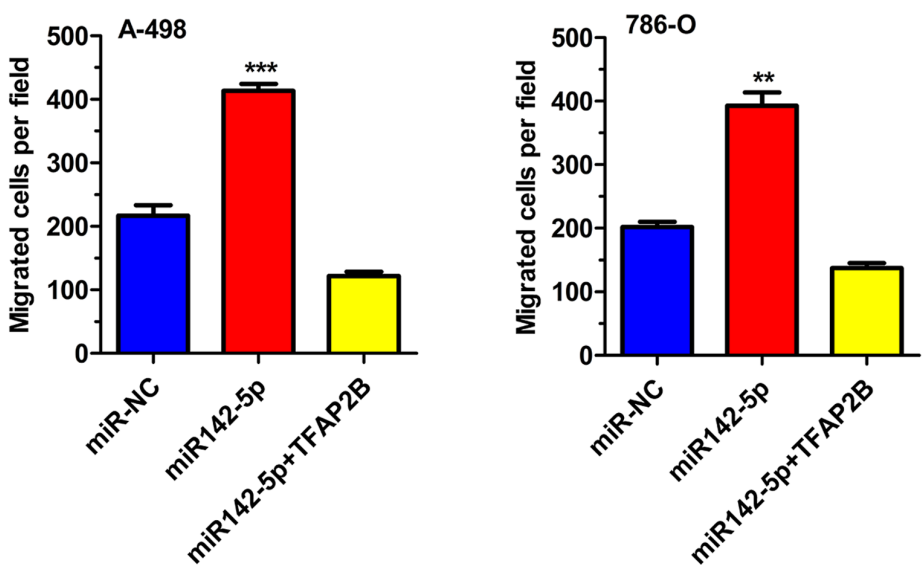

Figure 4. miR-142-5p promotes the migratory ability of renal cell carcinoma cells. (A) Representative images of Transwell migration assay in 786-O and A-498 cells overexpression miR-142-5p alone or in combination with TFAP2B overexpression. (B) Quantification of the migrated cells per field presented in (A) Data are presented as the mean $\pm \mathrm{SD} ;{ }^{* *} \mathrm{P}<0.01$ and ${ }^{* * * *} \mathrm{P}<0.001$ vs. miR-NC. miR, microRNA; NC, negative control; TFAP2B, transcription factor AP- $2 \beta$.

of ccRCCs by targeting BTG anti-proliferation factor 3. These results revealed the importance of the miR-142-5p in ccRCC development.

$T F A P 2 B$ expression has been previously detected in embryonic renal tissues and was found to be important for kidney developmentinmice(12).In2004,immunohistochemical evidence first suggested that transcription factor AP-2 may play a role in carcinogenesis (21). Furthermore, using comprehensive bioinformatic analyses, RT-qPCR and immunohistochemistry, downregulation of TFAP $2 B$ has been found to be important not only for normal renal development and epithelial differentiation but also for mesenchymal/adipogenic transdifferentiation and pluripotent mesenchymal stem cell-like differentiation (22). To explore the regulation network of the TFAP $2 B$ in renal cancer, the current study also analyzed the interaction partners of $T F A P 2 B$. The results with STRING software indicated that p53, the well-known tumor suppressor gene, might be important for the downstream and upstream regulation of $T F A P 2 B$ due to their interaction, which required further experiments to be confirmed. However, more experiments should be conducted to confirm this hypothesis in the future.

There are some limitations in the present study, such as lack of rescue experiments, interference experiments of $T F A P 2 B$ in cell lines, the limited number of ccRCC cell lines studied for functional assessment of the effects observed and the lack of reproductivity in the western blotting assays in other ccRCC cell lines. Although the detailed regulatory mechanistic functions of $T F A P 2 B$ in driving cancer remain to be further explored, to the best of our knowledge, this study is the first to demonstrate $T F A P 2 B$ regulation by miR-142-5p in ccRCC. In conclusion, miR-142-5p/TFAP $2 B$ pathway in ccRCC is described for the first time, which may provide novel targets for ccRCC therapy.

\section{Acknowledgements}

Not applicable.

\section{Funding}

This research was supported by The Provincial Natural Science Foundation of Fujian (grant no. 2018D0022) and The Xiamen Science and Technology Guiding Program of China (grant no. 3502Z20189043).

\section{Availability of data and materials}

The datasets used and/or analyzed during the current study are available from the corresponding author on reasonable request. 


\section{Authors' contributions}

MZ, LZ, JF and YL designed and performed the experiments. BS, KY, FL, LY and ML performed the experiments. All authors critically revised the manuscript. JF and YL supervised the project. All authors read and approved the final manuscript.

\section{Ethics approval and consent to participate}

Not applicable.

\section{Patient consent for publication}

Not applicable.

\section{Competing interests}

The authors declare that they have no competing interests.

\section{References}

1. Hsieh JJ, Purdue MP, Signoretti S, Swanton C, Albiges L, Schmidinger M, Heng DY, Larkin J and Ficarra V: Renal cell carcinoma. Nat Rev Disease Primers 3: 17009, 2017

2. Siegel RL, Miller KD and Jemal A: Cancer statistics, 2020. CA Cancer J Clin 70: 7-30, 2020.

3. Cho E, Adami HO and Lindblad P: Epidemiology of renal cell cancer. Hematol Oncol Clin North Am 25: 651-665, 2011.

4. Makhov P, Joshi S, Ghatalia P, Kutikov A, Uzzo RG and Kolenko VM: Resistance to systemic therapies in clear cell renal cell carcinoma: Mechanisms and management strategies. Mol Cancer Ther 17: 1355-1364, 2018.

5. Bray F, Ferlay J, Soerjomataram I, Siegel RL, Torre LA and Jemal A: Global cancer statistics 2018: GLOBOCAN estimates of incidence and mortality worldwide for 36 cancers in 185 countries. CA Cancer J Clin 68: 394-424, 2018.

6. Tippu Z, Au L and Turajlic S: Evolution of renal cell carcinoma Eur Urol Focus S2405-S4569: 30383-30389, 2020.

7. Mennitto A, Verzoni E, Grassi P, Ratta R, Fucà G and Procopio G: Multimodal treatment of advanced renal cancer in 2017. Expert Rev Clin Pharmacol 10: 1395-1402, 2017.

8. Eckert D, Buhl S, Weber S, Jäger R and Schorle H: The AP-2 family of transcription factors. Genome Biol 6: 246, 2005.

9. Lüscher B, Mitchell PJ, Williams T and Tjian R: Regulation of transcription factor AP-2 by the morphogen retinoic acid and by second messengers. Genes Dev 3: 1507-1517, 1989.

10. Zeng YX, Somasundaram K and el-Deiry WS: AP2 inhibits cancer cell growth and activates p21WAF1/CIP1 expression. Nat Genet 15: 78-82, 1997.
11. Auman HJ, Nottoli T, Lakiza O, Winger Q, Donaldson S and Williams T: Transcription factor AP-2gamma is essential in the extra-embryonic lineages for early postimplantation development. Development 129: 2733-2747, 2002.

12. Moser M, Pscherer A, Roth C, Becker J, Mücher G, Zerres K, Dixkens C, Weis J, Guay-Woodford L, Buettner R and Fässler R: Enhanced apoptotic cell death of renal epithelial cells in mice lacking transcription factor AP-2beta. Genes Dev 11: 1938-1948, 1997.

13. Fu L, Shi K, Wang J, Chen W, Shi D, Tian Y, Guo W, Yu W, Xiao X, Kang T, et al: TFAP2B overexpression contributes to tumor growth and a poor prognosis of human lung adenocarcinoma through modulation of ERK and VEGF/PEDF signaling. Mol Cancer 13: 89, 2014.

14. Konta T, Ichikawa K, Suzuki K, Kudo K, Satoh H, Kamei K, Nishidate E and Kubota I: A microarray analysis of urinary microRNAs in renal diseases. Clin Exp Nephrol 18: 711-717, 2014.

15. Wong NW, Chen Y, Chen S and Wang X: OncomiR: An online resource for exploring pan-cancer microRNA dysregulation. Bioinformatics 34: 713-715, 2018.

16. Livak KJ and Schmittgen TD: Analysis of relative gene expression data using real-time quantitative PCR and the 2(-Delta Delta C(T)) method. Methods 25: 402-408, 2001.

17. Liu S, Xiao Z, Ai F, Liu F, Chen X, Cao K, Ren W, Zhang X, Shu P and Zhang D: miR-142-5p promotes development of colorectal cancer through targeting SDHB and facilitating generation of aerobic glycolysis. Biomed Pharmacother 92: 1119-1127, 2017.

18. Wang Z, Liu Z, Fang X and Yang H: MiR-142-5p suppresses tumorigenesis by targeting PIK3CA in non-small cell lung cancer. Cell Physiol Biochem 43: 2505-2515, 2017.

19. Cheng D, Li J, Zhang L and Hu L: miR-142-5p suppresses proliferation and promotes apoptosis of human osteosarcoma cell line, HOS, by targeting PLA2G16 through the ERK1/2 signaling pathway. Oncol Lett 17: 1363-1371, 2019.

20. Liu L, Liu S, Duan Q, Chen L, Wu T, Qian H, Yang S, Xin D, He Z and Guo Y: MicroRNA-142-5p promotes cell growth and migration in renal cell carcinoma by targeting BTG3. Am J Transl Res 9: 2394-2402, 2017.

21. Oya M, Mikami S, Mizuno R, Miyajima A, Horiguchi Y, Nakashima J, Marumo K, Mukai M and Murai M: Differential expression of activator protein-2 isoforms in renal cell carcinoma. Urology 64: 162-167, 2004.

22. Tun HW, Marlow LA, von Roemeling CA, Cooper SJ, Kreinest P, Wu K, Luxon BA, Sinha M, Anastasiadis PZ and Copland JA: Pathway signature and cellular differentiation in clear cell renal cell carcinoma. PLoS One 5: e10696, 2010.

(7) $\odot$ This work is licensed under a Creative Commons Attribution-NonCommercial-NoDerivatives 4.0 International (CC BY-NC-ND 4.0) License. 